\title{
Bereaved Parents Health Status During the First 6 months After Their Child's Death
}

\author{
Nancy Dias, Debra Brandon, Joan E. Haase, Paula Tanabe
}

\section{Introduction:}

According to the CDC, 23,215 infants and an additional 9,000 children aged between 1 to 14 years died in 2014. ${ }^{1}$ The death of a child can completely transform the lives of the parents and can negatively impact their health. ${ }^{2,3}{ }^{4}$ However, there are discrepancies in the literature regarding the health of parents after their child's death. Death rates are highest among parents in the first 3 years after a child's death. The mortality rates for bereaved parents is 2 to 4 times higher when compared to non-bereaved parents. ${ }^{5}$ In contrast, some studies indicate that bereaved parents' mortality and morbidity rates are no different from the general population of parents. ${ }^{6}$ Mortality rates of bereaved parents monitored up to 19 years after a child's death showed no increase when compared to nonbereaved parents. ${ }^{6}$

Similar discrepancies are documented on bereaved parents' morbidity data. Higher incidences of physical and mental conditions such as cancer, cardiac illnesses, anxiety, depression, and post-traumatic stress symptoms were reported in bereaved parents when compared to non-bereaved parents. ${ }^{7-12}$ Hospitalizations, and medication changes are also highest in the first 6 months following a child's death. ${ }^{13}$ At 5 years after a child's death, mental distress and trauma is 2 to 3 times higher when compared to the general population. ${ }^{14}$ Long-term negative health outcomes are documented as late as 35 years after a child's death. ${ }^{15}$ Moreover, parents experience a decrease in their social interactions and some experience negative changes in relationships with others including their spouse. ${ }^{13,16}$ Alternately, some studies reported no differences in the risk for stroke ${ }^{17}$, myocardial infarction ${ }^{10}$, cancer survival, ${ }^{6,18}$ and frequency/duration of hospitalization ${ }^{19}$ between bereaved and non-bereaved parents. Despite these discrepancies, most of the research indicates that all aspects of a parent's health including their physical, mental, and social health are affected after a child's death.

Several factors contribute to the discrepancies in research on bereaved parents' health: variations in study methods (retrospective or prospective), sample sizes (small to large population based studies), deceased child's age (newborn to adult child), time since death (3 months to 35 years), parent's age (young to older adult), and psychometric properties of assessment tools (standardized established tool or self-report survey).$^{20}$ In addition, 
most studies that report no difference between the bereaved and non-bereaved parents were conducted outside the United States including Denmark, ${ }^{10,12}$ Sweden, ${ }^{21}$ and Israel. $., 6,22$ These discrepancies may reflect the morbidity and mortality of the different geographical locations and the cultural attitudes related to death and dying in that region. These variations continue to fuel the debate on whether or not a child's death influences bereaved parents' health.

In the United States, there is limited and conflicting data on the health of bereaved parents after losing a child. Birenbaum and colleagues found bereaved parents' health is not affected after their child's death. ${ }^{23}$ In contrast, Youngblut found bereaved parents' health may be "at risk" after losing their child; specifically they had changes in behaviors such as eating, sleeping, smoking, drinking alcohol, as well as changes in body weight, blood pressure, and blood sugar that could serve as health risk indicators in the first year following a child's death. ${ }^{13}$

To better understand the bereaved parents' health affects after their child's death, it is essential to gather enough data to address the following unanswered questions: When do adverse changes in health become evident in these parents? Can assessment tools identify at risk parents? What is the appropriate time to administer the assessment tools? Is it feasible for parents to fill out these assessment tools, considering their emotional status after their child's death? This information can help determine the need for and the timing of health screening, so that multi-disciplinary intervention programs with a focus on health promotions and disease preventive measures for bereaved parents can be designed and tested.

The purpose of this study was to assess bereaved parents' physical, sleep, mental, and social health with a goal to identify early health risk indicators during the first 6 months after their child's death. To understand which aspects of bereaved parents' health is most affected, this study used established and quantifiable health survey outcome measures recommended by the National Institute of Health, the Patient-Reported Outcomes Measurement Information System (PROMIS®) scales to assess all aspects of bereaved parents’ physical, mental, and social health. Additionally, since there is ample evidence that bereaved parents sleep is negatively impacted, ${ }^{24,25}$ we also included a sleep assessment scale (PROMIS®, sleep). To evaluate the timeframes for changes in bereaved parents' health, we measured health risk indicators across 2 time-points ( 3 and 6 months) after their child's death. This time frame is the most stressful time after their child's death ${ }^{13,26}$ and will provide data on the early indicators of poor health, which would allow for early assessment and intervention for parents exhibiting high risk criteria. Screening 
programs and early intervention for bereaved parents will guide the design of bereavement programs with a focus on health promotion and disease prevention, thus improving long-term health outcomes of bereaved parents. ${ }^{26}$

\section{Methods}

\section{Design}

We used a prospective case-based study approach to assess bereaved parents' health and bereavement challenges during the first 6 months after their child's death. Each case included parent dyads (mother and father) of children below 12 years who had died of any life-threatening illness. Individual interviews were conducted and parents were also asked to complete 5 health surveys and a demographics form. Interviews were done either through home-visits or via a telephone call. Demographic data and health surveys were administered via an email link. Additionally, a paper-based health survey was also administered. A paper format was used instead of digital due to copyright restrictions for that specific survey. Details of all health surveys are presented in the measures section. This manuscript presents all health survey data that assessed the parents' physical, sleep, mental, and social health.

\section{Sample and Setting.}

Approval for the study was obtained from the institutional review board for human subjects research. Informed written consent was obtained from all subjects prior to data collection.

Five cases were recruited through the palliative care program of an academic tertiary medical center in the Southeast region of the United States. This palliative care program offers a comprehensive, family centered palliative and end-of-life care for children with complex chronic or life-threatening conditions. Parents for additional three cases were recruited by word of mouth through nursing colleagues who personally knew the bereaved parents. While an attempt was made to recruit using support groups (Compassionate Friends, Kinder Mourn, and Kids Path), only one parent responded to a letter sent by the support group coordinator on behalf of the PI, but was unreachable for subsequent follow up. Thus, no participants were recruited using this method. Finally, a total of 8 cases were recruited within 9 months.

Eligible participants were parents $\geq 18$ years old who spoke and read English, and had experienced the death of a child below 12 years within the last two months from a life-threatening illness. Parents were excluded if 
they were $<18$ years of age at the time of their child's death because of different developmental needs of adolescent parents. Parents whose child died following a traumatic injury were also excluded, because traumatic deaths are associated with higher risk for complicated parental grief given the guilt associated with parents' actual or perceived role in the child's death. ${ }^{27,28}$ In addition, parents who experienced a fetal loss were excluded because of key differences in the grief needs of parents who experience fetal loss..$^{29,30}$

We used a convenience sampling strategy to recruit eligible parents. Parents who met study eligibility criteria were contacted via either a letter or phone call from a palliative care team member. The letter included a toll free opt-out telephone number. To further assess parents' willingness to participate in the study, parents who did not opt out were contacted by phone by the first author, 2-3 weeks after the letter was sent. Similarly, for parents who received phone calls by the palliative team member, their willingness to participate in the study was assessed during that phone call. Parents who were recruited by word of mouth, were contacted by an individual known to them to determine their willingness to participate in the study. Parents' who expressed their willingness to participate or learn more about the study were then contacted by the first author via a phone call to provide details of the study, obtain a verbal assent to send study material including consent form for their review, and obtain a potential date for data collection. To obtain child demographics and confirm study eligibility, verbal assent was obtained from parents who were recruited through the palliative care program, to view their child's medical record about illness specific data such as diagnoses, complications, and length of illness. For parents recruited via word of mouth, information about the child's illness was obtained from the parents.

A total of 17 eligible parent dyads were contacted. Among these, 8 parent dyads ( 8 mothers and 8 fathers) agreed to participate, enrolled in the study, and completed data collection at both time points. The recruitment rate was $47 \%$ with a $100 \%$ retention rate. Of the 9 parent dyads that did not participate, 7 were not reachable or did not respond to the telephone call. Of the remaining 2, one parent said that it would be too stressful to participate and another stated that the spouse was not willing to participate.

\section{Measures}

Demographic data included information about parent's age, gender, race, and income. 
Self-reported medical history included illness diagnosis (e.g. diabetes or hypertension) before and 6 months after their child's death, hospital admissions and emergency department visits, smoking and alcohol use, and use of bereavement services.

Self-Health perception scale (SHPS): Parent's health was assessed based on a scale from visual line scale 0 to 10 , with zero indicating worst health and 10 the best health possible. Parents identified a number, which best depicted their perception of their current overall health.

Validated Health Surveys

Patient-Reported Outcomes Measurement Information System [PROMIS] was used to measure parent's global, sleep, and social health and the Brief Symptom Inventory-18 (BSI-18) was used to assess their physical, sleep, mental, and social health.

PROMIS: All PROMIS surveys have established psychometric properties (Buysse et al., 2010; Castel et. al.; ref). PROMIS-global (version: 1.0) is a 10-item measure that assesses overall physical and mental health, including pain and fatigue. All PROMIS scale items are rated on a 5 point Likert scale. According to the PROMIS scoring manual (2014), the social and sleep scales T score of 50 is the average of the calibrated sample of people with chronic illnesses and the T score is provided with an error term (Standard Error) and for the PROMIS global, a $\mathrm{T}$ score of 50 is the average for the general population in the United States. All these scales have a standard deviation of 10. A higher PROMIS T score indicates better health when compared to the general population. The PROMIS-social (version: 8a) assesses the individual's perceived ability to participate in social activities. The PROMIS-sleep impairment scale (version-8a) includes 8 items that assess quality and ability to sleep.

The BSI-is an 18-item tool and is a multidimensional measure of mental distress that assesses specific psychiatric illness such as anxiety, depression, and somatization (Asner-Self, Schreiber, \& Marotta, 2006: Galdon et al., 2008) conditions predominantly observed in this population. The Cronbach's $\alpha$ for BSI is between $0.71-0.84$ (Merport \& Recklitis, 2012) with modest predictive and discriminate validity (Meachen, Hanks, Millis, \& Rapport, 2008). For the BSI-18, the paper version of the answer sheet was used to record the value (0-4) for each question in the corresponding line. The lines were arranged in three columns to correspond to the three BSI subscales: (1) 
Somatization, (2) Depression, and (3) Anxiety. The total values of each column were added to obtain raw scores for each subscale and the global (GSI) raw score was obtained by adding the sum of all subscales. The raw scores of each participant were plotted on the profile for community norms based on their gender (father's = male profile, mother's $=$ female profile) to obtain the T scores.

\section{Data Collection}

Data collection included both interview data and health survey data and occurred at 3 and 6 months after a child's death either in person at their home or via a telephone call. All parents completed health surveys prior to their qualitative interview. For parents who met in person with the first author, first completed the PROMIS surveys on an electronic device (iPAD or laptop) connected to parent specific REDCap electronic survey link or the BSI-18 (paper version). The SHPS was completed after all the health surveys were completed. The BSI-18 and the SHPS responses were then transferred to the REDCap database after the home visit was completed.

Parents who had telephone interviews PROMIS health survey REDCap link were sent via email. On the scheduled interview day, parents first completed the PROMIS health surveys, followed by the BSI-18 paper version and SHPS. Parents then orally reported responses to the BSI-18 and SHPS for entry into the REDCap database.

At the second time-point (6 months), the REDCap link was emailed a week prior to the scheduled interview to allow parents to complete the survey prior to the interview. If parents did not complete the measures prior to the scheduled interview, they were given time before their interview to complete the survey. No identifiable data were recorded on the paper versions to mainatian particpant privacy.

\section{Data Analysis}

PROMIS and BSI-18 health survey raw scores were converted to T- scores based on the T-score conversion table provided for each scale. The differences between each family's father's and mother's score was calculated and summarized at each time point. Each parent's change from 3 to 6 months was also calculated and summarized separately for each gender. Cliff's delta, which ranges from -1 to +1 , was used as a nonparametric measure of effect size. A positive Cliff's delta indicated that the father's score was more often larger than the mother's score; and, a negative Cliff's delta indicated the mother's score was more often larger than the father's 
score. The difference between the 6 months to 3 months change in the father's score and the change in the mother's score was also calculated and summarized. Using the change data for father and mother, Cliff's delta was calculated as the measure of effect size for the difference of changes.

For the self-health perception scale, individual parent scores at each time point and a difference from 3 months to 6 months is summarized. Additionally, the nonparametric Spearman rank correlation coefficient (rho) was used to estimate the associations between the self-health perception with the PROMIS and BSI subscales.

Data summaries and correlation coefficients were generated using SAS/STAT software, Version 9.4 of the SAS System for Windows. Copyright (C) 2002-2012 SAS Institute Inc. SAS and all other SAS Institute Inc. product or service names are registered trademarks or trademarks of SAS Institute Inc., Cary, NC, USA. Cliff's delta and the 95\% confidence intervals were calculated using R version 3.1.3 (2015-03-09) -- Copyright (C) 2015.

\section{Results}

Table 1 shows the participant and child demographics. Overall, parents were within the ages of 30 to 50 and had some racial diversity including Caucasians $(\mathrm{n}=10)$, African Americans $(\mathrm{n}=2)$, and Asians $(\mathrm{n}=4)$. The children's age ranged from 48 hours after birth up to 12 years of age at the time of death. All children (n=8) were diagnosed with a life-threatening illness including cancer $(n=3)$, congenital illness $(n=4)$, and prematurity $(n=1)$. All the parents had at least 1 year of college education and six of the eight parent dyads had a combined household income of 60,000 or greater. Most of the parents $(n=13)$ were employed with no change in job status at the 3 and 6 month time-points. Two mothers were homemakers and one mother who was employed prior to her child's death, was not working up to 6 months after her child's death. At the 6-month interview, this mother stated that she was soon going to take a new job. Most of the parents had at least one living child $(\mathrm{n}=14)$.

\section{Self-reported medical history:}

Based on the self-reported medical history data, four (25\%) of the 16 parents ( 8 mothers and 8 fathers) had new diagnosis within the 6-month period after their child's death. One mother and one father reported prediabetes and anxiety as new diagnoses at the 3-month time point and two mothers reported anxiety and sleep disorder as new diagnoses at the 6-month time point. One father was referred to a psychiatrist for further evaluation, but was not medically diagnosed with any condition. Self-reported smoking was unchanged, but two 
parents reported an increase in alcohol consumption after their child's death. The mother diagnosed with sleep disorder reported that she was taking medications to aid her sleep.

SHPS: Most parents $(\mathrm{n}=14,87.5 \%)$ rated their overall health at a 7 or 8 (See Figure 1) at both time points. While $43.75 \%$ of the parents (mothers $=4$, fathers $=3$ ) had an improved score at 6 months, the scores of more fathers than mothers were reduced $(n=4,25 \%$; mothers $=1$, fathers $=3)$ and more mothers than fathers had stable scores at both time points $(\mathrm{n}=5,31.25$; mothers $=3$, fathers $=2)$.

Health Surveys:

Table 2. summarizes the descriptive statistics of the PROMIS and BSI-18 health surveys for the mothers and fathers and the score changes from T1 to T2. On the PROMIS health measures, all parents exhibited health scores below the population mean for at least one area of health status. Social health was the most affected, with $100 \%$ of parents' social health scores below the population mean; the lowest score was 25.9 , which is three standard deviation below population mean $(\mathrm{Score}=50, \mathrm{SD}=10)$. Physical health scores were the least affected; yet around $40 \%$ parents had scores below population means.

On the BSI-18, one mother had a subscale score for depression above the cut off score of 63 at both 3 and 6 months and a global score of 63 at 6 months, thus warranting further clinical evaluation. Two mothers scored above 63 on at least one subscale at 3 months. Regarding fathers, one scored above 63 on the global score at 3 months and one scored above 63 on at least one subscale at both the time points. Most parent's scores (mothers $=43.75$, fathers $=37.5$ ) did not indicate the need for further mental health evaluation for specific disease diagnosis at either time points.

Within group comparisons, (Figure 3) include interpretations of the direction of average within gender changes. Higher PROMIS scores indicate better health and higher BSI scores indicate worse health; that is, an increase in PROMIS scores and a decrease in BSI scores from time 1 to time 2 indicate an improvement in health. On average, mothers showed improvement in PROMIS sleep t-scores, BSI somatization t-score, BSI depression tscore, BSI anxiety t-score and BSI global score t-score, though none of these improvements were statistically significant as seen by the inclusion of zero in the $95 \%$ confidence intervals. Mothers on average had worsening in the PROMIS physical t-score and the PROMIS social t-score. These confidence intervals also included zero and 
declines were not statistically significant changes as $p$-value were $<0.05$. Fathers, on average, improved in the PROMIS mental t-score, the PROMIS social t-score, the BSI somatization t-score, the BSI depression t-score, the BSI t-anxiety score and the BSI global score t-score. Only the $95 \%$ confidence interval of the BSI global score tscore lies completely below zero, indicating fathers' BSI global score was a statistically significant improvement.

Between group comparisons, (Table 3.) summarize the difference in scores between fathers and mothers, including the Cliffs delta and 95\% confidence interval. All 95\% confidence intervals were 0 , indicating the lack of a significant difference between father's and mother's scores. Nevertheless, the point estimate of the effect sizes can be used to design a larger study. At 3 months, the PROMIS physical, mental, social scores, and the BSI somatization subscale score had positive Cliff's delta, indicating that, in general, fathers' scores were larger (i.e. better) than mothers' scores. However, none of these differences were statistically significant, since the corresponding 95\% confidence intervals included a zero. Correspondingly, at 6 months, the PROMIS mental, sleep scores, the BSI depression, anxiety subscale scores, and the SHPS had positive Cliff's delta. Similar to the difference between fathers and mothers at 3 months, these effect sizes were not statistically significant at the $\alpha=0.05$ level. Spearman's correlations between the health surveys (PROMIS \& BSI-18) and SHPS were not strong (See Table 4.) as the spearman $r$ coefficient were not closer to 1 or -1 . Thus, parents' self-assessment of their health did not strongly associate with the objective health survey scores and were not statistically significant.

\section{Discussion:}

This is the first prospective study to evaluate bereaved parents' physical, mental, and sleep health prospectively during the most stressful time frame ( $1^{\text {st }} 6$ months $)$ after their child's death using standardized health surveys which, allow comparison of resulting scores to the general U.S. population health data (T scores). Previous research assessed bereaved parents' health during the first 13 months after their child's death, evaluated grief, mental health, and self-reported physical health. ${ }^{13,26}$ The importance of prospective and early assessment ( 3 and 6 months) in this study cannot be over-estimated because it allows for the early identification of potential health risks and thus early intervention.

This study supports the findings of previous studies that bereaved parents' health is susceptible to illnesses. Based on the self-reported medical history, it is significant to note that out of the 16 participants in this 
study, four participants had new diagnosis within the first 6 months. These findings underscore that bereaved parents' health is "at risk". ${ }^{13}$ Additionally the self-health report data highlights other related health issues such as increase in habits such as smoking or alcohol consumption and sleep deprivation that can lead to chronic illnesses and therefore deserves attention too. Thus, including assessment of change in habits or behaviors would be a necessary step towards health promotion and disease prevention. Moreover, the lack of correlation between the objective health surveys and parents' self-health perception indicates that bereaved parents may not be able to objectively assess their health due to their focus on their grief. ${ }^{31}$ Consequently, parents may delay accessing the health care system until presented with specific health condition, which emphasizes the need for the health care system to be proactive and invest in health promotion and preventive services for bereaved parents.

Consistent with other studies, our results indicate bereaved parents have at least one health concern during the first six months following their child's death. The sleep and social health data from this study confirm findings from a qualitative study which identified bed-time was the most difficult time for bereaved parents, and that parents' social interactions with family, friends, and community in general as negatively affected. ${ }^{25}$ Additionally, the potential lack of sleep ${ }^{32}$ and decline in the quality of social relationships ${ }^{33}$ may be precursors that affect other health outcomes including physical and mental health. Comparisons of three and six months' health scores identified that mothers had trends towards worsening in their physical and social health, also indicating a need for further examination of the relationship of social health to parents' physical health.

Comparison between mothers' and fathers' health concurred with prior studies, in which fathers fared better than mothers at six months. ${ }^{19}$ Although the Cliffs Delta (an indication of effect sizes) on the PROMIS sleep scale, the BSI scale, and the SHPS were all positive indicating that the change in the father's score was, in general, larger than the change in the mother's scores. Though these scores were not statistically significant, at the $\alpha=0.05$ level, these estimated effect sizes will be particularly useful for informing the sample size and power calculation for a future study.

Recruitment for this study was a long process, because it was difficult to reach out to parents immediately after their child died. Health care providers served as gate-keepers for this sensitive group of people, which while well intended, can hinder obtaining the important information needed to understand the needs, challenges, and 
health concerns of this population at the most critical time. ${ }^{34}$ The $100 \%$ retention rate in this study supports the assumption that bereaved parents become altruistic after their child's death and are willing to tell their story to help other bereaved parents. ${ }^{35}$ Most of the parents in this study also acknowledged their altruistic motivations to share their stories with the intent to help other bereaved parents. Although support groups may be an alternative recruitment venue, it also may provide access to a biased sample that is already reaching out for help. Therefore, using the hospital-based palliative care program was intentional to reach out to all parents and provide them with an opportunity to tell their story and assess their health.

Access to a hospitalized pediatric palliative care program however, provides parents of terminally ill children an opportunity to foster a relationship with a palliative team during the child's illness. This relationship can potentially have a positive effect on parents experience during the child's end-of-life and consequentially have a positive influence on their grief and health outcomes after their child's death. ${ }^{36}$ Ten of the 16 participants in this study were recruited through the palliative care program and possibly benefitted from the relationship developed with the palliative care team during the child's illness. Though, offering pediatric palliative care services by pediatric hospitals is on the rise, there are still many facilities that do not offer these services. ${ }^{37}$ This population may have different pre-death experiences, leading to different post-death grief and health outcomes.

This study has both strengths and limitations. Strengths include the prospective and longitudinal design, early assessment at 3 months, use of validated health measures, $100 \%$ retention, and comparisons between both fathers and mothers. Limitations include a small sample size; however, the case study design is suitable for smaller sample size studies. In addition, the sample was not representative of racial demographic. Despite the limitations, this study provides foundational data about bereaved parents' health to guide future longitudinal studies that address the limitation of a small sample size and a short time-frame ( $1^{\text {st }} 6$ months). Additional objective data that assesses the trajectory of bereaved parents over longer time-frames is essential to address concerns regarding bereaved parents' health.

\section{Conclusion}

Our study provides essential baseline empirical data to confirm that bereaved parents are indeed at risk for health problems. It is imperative that bereaved parents are made aware of the potential health consequences, and 
are provided with tools to monitor their health. Bereaved parents' health merits the attention of the healthcare system, specifically improvement in parental bereavement care services, including early risk assessment and interventions that target health promotion and prevention. 